\title{
SEMISIMPLE CLASSES AND UPPER-TYPE RADICAL CLASSES OF NARINGS
}

\author{
TERRY L. JENKINS AND DARYL KREILING
}

ABstract. In the universal class of associative rings every class $M$ of rings determines a unique upper radical class. It is shown that the same result is valid in the universal class of alternative rings but is not necessarily true in the universal class of narings. The latter class does however determine a type of upper radical.

1. Introduction. In $[4$, p. 1165] Yu-Lee Lee has shown that in the universal class of associative rings every class $M$ of rings determines an upper radical class. The purpose of this paper is to point out the validity of the same result in the universal class of alternative rings and to show that in the universal class of narings (not necessarily associative rings) a class $M$ need not determine a unique upper radical class. This leads to the concept of an upper-type radical class determined by a class of narings. The example which is presented also shows that in the universal class of narings the intersection of semisimple classes need not be a semisimple class.

The following lemmas will be used.

Lemma 1 [2, p. 19]. If the class $M$ of narings satisfies condition

Each nonzero ideal of an $M$-naring maps homomorphically onto some nonzero $M$-naring,

then

(1) The class $U(M)=\{A \mid A$ has no nonzero homomorphic images in $M\}$ is a radical class.

(2) Every $M$-naring is $U(M)$-semisimple.

(3) If $P$ is a radical class of narings such that $M$ is contained in the class of $P$-semisimple narings, then $P \subseteq U(M)$.

Because of the maximality of $U(M)$ among the radical classes for which each member of $M$ is semisimple, $U(M)$ is called the upper radical class determined by $M$.

LemMa $2[1$, p. 600]. If $P$ is a radical class in the universal class of alternative rings, then for any alternative ring $R$ and any ideal $I$ of $R$, the $P$ radical of $I$ is an ideal of $R$.

Presented to the Society, August 26, 1970; received by the editors February 12, 1970.

AMS 1969 subject classifications. Primary 1330, 1630, 1750; Secondary 1632, 1760.

Key words and phrases. Alternative rings, upper radical class, semisimple rings, upper-type radical classes, narings. 
Lemma 3. With $U(M)$ as defined in (1), $U(M)$ contains the simple naring $R$ if and only if $M$ does not contain $R$.

Proof. It is clear that $R \in U(M)$ implies $R \notin M$. Conversely, suppose the simple naring $R$ is not in $M$. Since the only nonzero homomorphic image of $R$ is an isomorphism, $R \in U(M)$.

\section{Upper radical classes in alternative rings and narings.}

THEOREM 1. In the universal class of alternative rings every class $M$ determines an upper radical class.

Proof. The proof is essentially that of $[4$, Theorem 3$]$ and will not be repeated here. We point out only that Lemma 2 is applied instead of the corresponding result for associative rings.

To see that Theorem 1 does not hold in the universal class of narings we consider the following example. Let $R$ be the naring generated over $G F(2)$ by the symbols $\{u, v, w\}$ satisfying the relations $u^{2}=0, u v=v u=u, u w=w u=v w=w v=v^{2}=v$ and $w^{2}=w[3, \mathrm{p}$. 1116]. It is clear that $I=\{0, u, v, u+v\}$ is the only proper ideal of $R$ and that $J=\{0, u\}$ is the only proper ideal of $I$. Also we observe that $R / I \cong I / J \cong G F(2)$ and that $J$ is a simple zero ring.

Let $M=\{R\}$. We complete $M$ to classes $M_{i}, i=1,2,3$, each satisfying ( $\mathrm{S} 1$ ) by including in $M_{i}$ nonzero homomorphic images of each ideal of narings in $M_{i}$. There is no loss of generality in including $R$ in each such class since $R$ will be semisimple relative to the resulting upper radical class. The possible classes are $M_{1}=\{R, I, J\}, M_{2}$ $=\{R, R / I \cong I / J\}$ and $M_{3}=\{R, I, R / I \cong I / J, J\}$.

Let $P$ be any radical class such that $R$ is in $S P$, the class of $P$ semisimple narings. Then either $M_{1} \subseteq S P$ or $M_{2} \subseteq S P$ so either $P \subseteq U\left(M_{1}\right)$ or $P \subseteq U\left(M_{2}\right)$. Hence, the only possibilities for an upper radical class determined by $M$ are $U\left(M_{1}\right)$ or $U\left(M_{2}\right)$. However, since the simple naring $J \in M_{1}$, then $J \in U\left(M_{1}\right)$ but $J \notin M_{2}$ so $J \in U\left(M_{2}\right)$ by Lemma 3 . Therefore $U\left(M_{2}\right) \Phi U\left(M_{1}\right)$. Similarly the simple naring $I / J \in M_{2}$ so $I / J \notin U\left(M_{2}\right)$ but $I / J \notin M_{1}$ so $I / J \in U\left(M_{1}\right)$. Therefore $U\left(M_{1}\right) \nsubseteq U\left(M_{2}\right)$. Hence, there is no upper radical class determined by $M=\{R\}$.

It is known that in the universal class of associative rings and in the universal class of alternative rings the intersection of semisimple classes is a semisimple class. The radical classes $U\left(M_{1}\right)$ and $U\left(M_{2}\right)$ enable us to show that in the universal class of narings this need not be the case.

Let $M_{1}^{*}$ and $M_{2}^{*}$ denote the semisimple classes of the radical classes $U\left(M_{1}\right)$ and $U\left(M_{2}\right)$ respectively. Suppose $M_{1}^{*} \cap M_{2}^{*}$ is a semisimple 
class. Since $R \in M_{1}^{*} \cap M_{2}^{*}$ and $I$ is an ideal of $R$, either $I$ or its only other nonzero homomorphic image $I / J$ must be in $M_{1}^{*} \cap M_{2}^{*}$. However, as observed above, $I / J \in U\left(M_{1}\right)$ so $I / J \notin M_{1}^{*} \cap M_{2}^{*}$. Thus $I \in M_{1}^{*} \cap M_{2}^{*}$, so its only proper ideal $J$, which is simple, must be in $M_{1}^{*} \cap M_{2}^{*}$. Again as observed above $J \in U\left(M_{2}\right)$ so $J \notin M_{1}^{*} \cap M_{2}^{*}$. This contradiction shows that $M_{1_{\mathrm{j}}}^{*} \cap M_{2}^{*}$ is not a semisimple class.

3. Upper-type radical classes of narings. The failure of a class $M$ of narings to determine a unique upper radical class leads to the concept of an upper-type radical class determined by $M$. To this end the following construction is helpful. Let $M$ be a nonempty class of narings and define $D_{1}(M)=\{I: I$ is a homomorphic image of an ideal of some $R \in M\}$. Assuming $D_{n}(M)$ has been defined, let $D_{n+1}(M)$ $=\left\{I: I\right.$ is a homomorphic image of an ideal of some $\left.R \in D_{n}(M)\right\}$. By the principle of mathematical induction $D_{n}(M)$ is defined for all natural numbers $n$. Let $M^{\prime}=\mathrm{U}_{n=1}^{\infty} D_{n}(M)$.

THEOREM 2. With $M^{\prime}$ as defined above, $M^{\prime}$ satisfies property (S1) and thus determines an upper radical class $U\left(M^{\prime}\right)$.

Proof. If $R \in M^{\prime}$, then $R \in D_{n}(M)$ for some natural number $n$. Hence, for any ideal $I$ of $R$ every homomorphic image of $I$ is in $D_{n+1}(M)$ and thus also in $M^{\prime}$. That $M^{\prime}$ determines an upper radical class $U\left(M^{\prime}\right)$ follows from Lemma 1.

At each step of the above construction of $M^{\prime}$ all homomorphic images of ideals of narings in the previously defined class were included. To satisfy property (S1) all that is required is at least one such nonzero homomorphic image. In this sense $M^{\prime}$ is the largest class to be constructed from $M$ which satisfies (S1) unless some arbitrary narings, unrelated to $M$ in terms of property (S1), are included. This largeness is transformed into smallness when the upper radical class is formed. We, therefore, make the following definition.

Definition 1 . Let $M$ be a class of narings. The radical class $U\left(M^{\prime}\right)$ constructed above is called the minimal upper-type radical class determined by $M$.

Definition 2. Let $M^{\prime}$ be the class of narings constructed above from the class $M$, let $P$ be a radical class and let $S P$ be the class of $P$-semisimple narings. If $U\left(M^{\prime}\right) \subseteq P$ and $M \subseteq S P$ we call $P$ an uppertype radical class determined by $M$.

From Definition 1 and Definition 2 it is clear that the minimal upper-type radical class determined by $M$ is an upper-type radical class determined by $M$ and is minimal among such radical classes.

With $R, I$ and $J$ the narings considered in $\S 3$ and with $M=\{R\}$, 
$M_{1}=\{R, I, J\}, M_{2}=\{R, R / J \cong I / J\}$ and $M_{3}=\{R, I, R / I \cong I / J, J\}$, the following observations are made. $U\left(M_{3}\right)$ is the minimal uppertype radical class determined by $M$, and both $U\left(M_{1}\right)$ and $U\left(M_{2}\right)$ are upper-type radical classes determined by $M$.

Let $M$ be a class of narings. An upper-type radical class determined by $M$ can be constructed as follows. This construction differs from that of $M^{\prime}$ to allow the use of the axiom of choice.

For each nonzero ideal $I$ of a naring $R$ we choose a nonempty set $S_{I}$ of nonzero homomorphic images of $I$ and define $H_{1}(R)=\left\{J \mid J \in S_{I}\right.$ for some ideal $I$ of $R\}$. Assuming $H_{n}(R)$ has been defined, for each naring $I$ of $H_{n}(R)$ and for each nonzero ideal $J$ of $I$, we choose a nonempty set $S_{J}$ of nonzero homomorphic images of $J$ and define $H_{n+1}(R)=\left\{K \mid K \in S_{J}\right.$ for some $J$ an ideal of some $\left.I \in H_{n}(R)\right\}$. Finally let $H(R)=\bigcup_{n=1}^{\infty} H_{n}(R)$.

Now let $M$ be a class of narings and let $M^{\prime \prime}=\{I \mid I \in H(R)$ for some $R \in M\}$.

THEOREM 3. With $M^{\prime \prime}$ as defined above $M^{\prime \prime}$ satisfies condition (S1) and hence determines an upper radical class $U\left(M^{\prime \prime}\right)$. Moreover, $U\left(M^{\prime \prime}\right)$ is an upper-type radical class determined by $M$.

Proof. Let $R \in M^{\prime \prime}$ and let $I$ be a nonzero ideal of $\mathrm{R}$. Then $R \in H(A)$ for some $A \in M$ so that $R \in H_{n}(A)$ for some $A \in M$ and some natural number $n$. Hence, from the construction of $H(A)$, some nonzero homomorphic image of $I$ is in $H_{n+1}(A)$ and so also in $H(A)$. That is, some nonzero homomorphic image of $I$ is in $M^{\prime \prime}$ and so $M^{\prime \prime}$ satisfies condition (S1). It follows by Lemma 1 that $M^{\prime \prime}$ determines an upper radical class $U\left(M^{\prime \prime}\right)$.

It is clear from the constructions that $M^{\prime \prime} \subseteq M^{\prime}$ so that $U\left(M^{\prime}\right)$ $\subseteq U\left(M^{\prime \prime}\right)$. It remains only to show that the class $M$ is contained in the class $S U\left(M^{\prime \prime}\right)$ of $U\left(M^{\prime \prime}\right)$-semisimple narings. $S U\left(M^{\prime \prime}\right)$, being a semisimple class, satisfies $[2$, p. 17] condition:

If $R$ is a naring such that every nonzero ideal of $R$ maps

(S2) homomorphically onto a nonzero naring in $S U\left(M^{\prime \prime}\right)$ then $R \in S U\left(M^{\prime \prime}\right)$.

From the construction of $M^{\prime \prime}$, if $R \in M$, then each nonzero ideal $I$ of $R$ maps homomorphically onto some nonzero naring in $M^{\prime \prime} \subseteq S U\left(M^{\prime \prime}\right)$. Hence, by (S2), $R \in S U\left(M^{\prime \prime}\right)$ as desired.

\section{REFERENCES}

1. T. Anderson, N. Divinsky and A. Sulinski, Hereditary radicals in assooiative and alternative rings, Canad. J. Math. 17 (1965), 594-603. MR 31 \#215. 
2. A. G. Kuroš, Radicals of rings and algebras, Mat. Sb. 33 (75) (1953), 13-26. (Russian) MR 15, 194.

3. W. G. Leavitt and E. P. Armendariz, Nonhereditary semisimple classes, Proc. Amer. Math. Soc. 18(1967), 1114-1117. MR 36 \#3838.

4. Yu-lee Lee, On the construction of upper radical properties, Proc. Amer. Math. Soc. 19(1968), 1165-1166. MR $38 \# 178$.

UNIVERSITY OF WYOMING, LARAMIE, WYOMING 82070

WESTERN ILLINOIS UNIVERSITY, MACOMB, ILLINOIS 61455 